\title{
A hybrid process combining homogeneous catalytic ozonation and membrane distillation for wastewater treatment
}

\author{
Yong Zhang a, b, Peng Zhao ${ }^{c}$, Jie Li ${ }^{\text {a, b }}$, Deyin Hou ${ }^{\text {a, b }}$, Jun Wang ${ }^{\text {a, b, * , Huijuan Liu }}{ }^{\text {a, b }}$ \\ a Beijing Key Laboratory of Industrial Wastewater Treatment and Resource Recovery, Research Center for Eco-Environmental Sciences, Chinese Academy of \\ Sciences, Beijing, China \\ ${ }^{\mathrm{b}}$ Key Laboratory of Drinking Water Science and Technology, Research Center for Eco-Environmental Sciences, Chinese Academy of Sciences, Beijing, China \\ ${ }^{\mathrm{c}}$ Department of Water Resources and Environmental Engineering, East China Institute of Technology, Nanchang, China
}

\section{H I G H L I G H T S}

- For the first time, homogeneous catalytic ozonation and membrane distillation were coupled in a membrane reactor.

- Organics and salts were efficiently removed and the homogenous catalyst was almost 100\% recovered simultaneously.

- Membrane fouling was effectively mitigated compared to MD process.

\section{A R T I C L E I N F O}

\section{Article history:}

Received 18 April 2016

Received in revised form

12 June 2016

Accepted 20 June 2016

Available online 30 June 2016

Handling Editor: Shane Snyder

\section{Keywords:}

Homogeneous catalytic ozonation

Membrane distillation

Membrane reactor

Saline organic wastewater
G R A P H I C A L A B S T R A C T

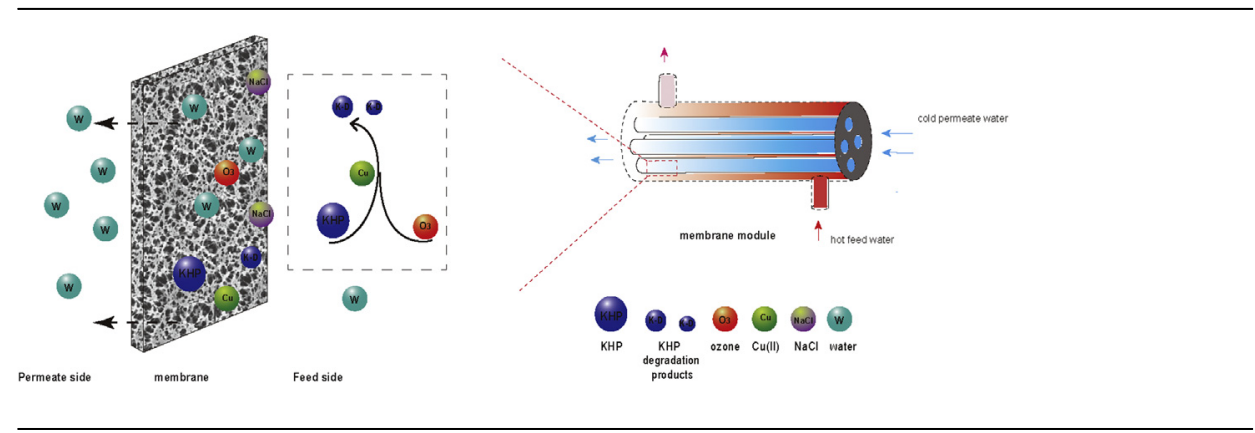

\begin{abstract}
A B S T R A C T
A novel catalytic ozonation membrane reactor (COMR) coupling homogeneous catalytic ozonation and direct contact membrane distillation (DCMD) was developed for refractory saline organic pollutant treatment from wastewater. An ozonation process took place in the reactor to degrade organic pollutants, whilst the DCMD process was used to recover ionic catalysts and produce clean water. It was found that $98.6 \%$ total organic carbon (TOC) and almost $100 \%$ salt were removed and almost $100 \%$ metal ion catalyst was recovered. TOC in the permeate water was less than $16 \mathrm{mg} / \mathrm{L}$ after $5 \mathrm{~h}$ operation, which was considered satisfactory as the TOC in the potassium hydrogen phthalate (KHP) feed water was as high as $1000 \mathrm{mg} / \mathrm{L}$. Meanwhile, the membrane distillation flux in the COMR process was $49.8 \%$ higher than that in DCMD process alone after $60 \mathrm{~h}$ operation. Further, scanning electron microscope images showed less amount and smaller size of contaminants on the membrane surface, which indicated the mitigation of membrane fouling. The tensile strength and FT-IR spectra tests did not reveal obvious changes for the polyvinylidene fluoride membrane after $60 \mathrm{~h}$ operation, which indicated the good durability. This novel COMR hybrid process exhibited promising application prospects for saline organic wastewater treatment.
\end{abstract} ๑) 2016 Elsevier Ltd. All rights reserved.

\footnotetext{
* Corresponding author. 18 Shuangqing Road, Haidian District, Beijing, 100085, China.
}

E-mail address: junwang@rcees.ac.cn (J. Wang).

\section{Introduction}

Ozone, as an important oxidant agent, had been considered for numerous applications in water and wastewater treatment (Gottschalk et al., 2000; Beltrán, 2004). It can react with compounds directly or through an indirect approach which generates 
hydroxyl radicals or metal-complexes (Pines and Reckhow, 2002; Beltran et al., 2005; Nawrocki and Kasprzyk-Hordern, 2010). In many cases, the hydroxyl radicals or metal-complexes approach had much greater reaction rate constants than direct ozonation, which were considered to be a promising method to improve the ozonation efficiency. (Pines and Reckhow, 2002; Beltran et al., 2005; Buffle et al., 2006). An important type of indirect process was catalytic ozonation which can be either homogeneous (catalyzed by transition metal ions), or heterogeneous (catalyzed by solid catalysts). The homogeneous catalysts usually showed good ozonation efficiency and low cost. However, the catalytic ions were difficult to recover which increases the process cost and introduces potential secondary pollution (Nawrocki and Kasprzyk-Hordern, 2010).

Membrane processes, such as nanofiltration (NF), reverse osmosis, and membrane distillation (MD) were reported with excellent performance for metal ion removal (El-Bourawi et al., 2006; Wachinski, 2013). MD process, in particular, only allows vapor molecules to transport across the membrane with almost $100 \%$ ion rejection. In addition, the membrane fouling was much less severe compared to those pressure-driven membrane processes (El-Bourawi et al., 2006). Further, MD process was also able to utilize low-grade waste and alternative energy sources, such as solar power (Shim et al., 2015). All the merits above made MD an attractive approach for homogenous catalyst recovery.

A large number of research investigated employing membrane process, including MD process, for catalyst recovery (Mozia and Morawski, 2006; Mozia et al., 2006; Choo et al., 2008; Benotti et al., 2009; Mozia, 2010; Mozia et al., 2010, 2014; Leong et al., 2014; Qu et al., 2014). Among these, photocatalytic membrane reactors (PMRs) were widely studied. In a $\mathrm{PMR}, \mathrm{TiO}_{2}$ particles (one type of photocatalyst) were commonly used to suspend in reactors, whilst the membrane processes, such as microfiltration, UF, NF and MD, were used to separate catalysts. The use of PMRs shortened the process route for catalyst recovery, reduced the size of installation, and also enabled the continuous production with low energy consumption (Mozia, 2010). In this regard, PMRs were considered to be a promising technology in natural organic matter (Choo et al., 2008), pharmaceuticals and endocrine disrupting compounds removal (Benotti et al., 2009), municipal wastewater (Mozia et al., 2014), and dye wastewater treatment (Mozia et al., 2006, 2009, 2010). Mozia et al (Mozia et al., 2014). compared two types of PMRs (utilizing UF or direct contact membrane distillation (DCMD)) for treating primary and secondary effluents of municipal wastewater plant. Photocatalysis-DCMD system offered better performance, with much lower flux decline and less membrane fouling. The total organic carbon (TOC) removal efficiency was significantly higher and the organic carbon in permeate water was less than $0.5 \mathrm{mg} / \mathrm{L}$. Another work of Mozia et al (Mozia et al., 2006). compared the above two systems for dye wastewater treatment. The photocatalysis-DCMD system also exhibited better permeate water quality and less membrane fouling, with $80 \%$ TOC removed in permeate stream.

The aforementioned examples confirmed that the photocatalysis-DCMD process using heterogeneous catalysts can effectively remove organic contaminants from aqueous solutions. It was postulated that the use of homogenous catalysts might bring in excellent performance as heterogeneous ones. In this regard, we proposed to combine homogeneous catalytic ozonation and DCMD for a hybrid catalytic ozonation membrane reactor (COMR) process. In this process, DCMD is used as catalyst separator and clean water producer, just similar to that of photocatalysis-DCMD process. Catalysts employed here are metal ions instead of the solid ones. The ionic catalysts were difficult to remove in conventional processes, however when combined with a MD process, an easy removal can be envisioned. Although the concept appeared to be promising, the COMR had been barely investigated in previous studies.

In this work, the feasibility of utilizing COMR for water treatment was evaluated in terms of the permeate water quality, catalyst recovery efficiency, MD flux and membrane fouling behavior. The membrane properties before and after operation were also evaluated to assess the material stability.

\section{Experimental}

\subsection{Wastewater}

The model saline wastewater used in this work contained $\mathrm{NaCl}$ and KHP with concentrations ranging from 200 to $1000 \mathrm{mg} / \mathrm{L}$. KHP was a non-volatile and stable refractory pollutant, it represented a class of endocrine disrupting compounds which is a major concern in environment (Heng et al., 2007; Kit Chan et al., 2012). Further, the addition of $\mathrm{NaCl}$ was to evaluate the rejection performance of COMR on monovalent ions. To avoid the sedimentation of catalysts, feed water was adjusted to $\mathrm{pH}$ value of 2 by adding certain amount of sulfuric acid.

\subsection{Membrane and membrane module}

Polyvinylidene fluoride (PVDF) hollow fiber membranes were prepared in-house via the dry-jet wet spinning technique. The detailed hollow fiber fabrication protocol can be found in literature (Hou et al., 2012). The dope solution comprised 15 wt\% PVDF (Solef ${ }^{(\mathbb{P}}$ PVDF1010, Solvay, Belgium) and $85 \mathrm{wt} \%$ dimethylacetamide. The resultant PVDF hollow fiber had an outer diameter of $1.36 \mathrm{~mm}$ and an inner diameter of $1.10 \mathrm{~mm}$. The average pore size was $168 \mathrm{~nm}$ and the pore size distribution and membrane section morphology can be seen in Fig. S1. The contact angle between membrane and water was $95.8^{\circ}$, and the porosity was $66 \%$. Each membrane module contained 20 PVDF hollow fibers with an effective area of $0.00854 \mathrm{~m}^{2}$.

\subsection{COMR hybrid process}

A laboratory-scale COMR set-up was presented in Fig. 1, which consisted of a PVDF hollow fiber membrane module, an ozone generation and injection system, a feed water circulation system

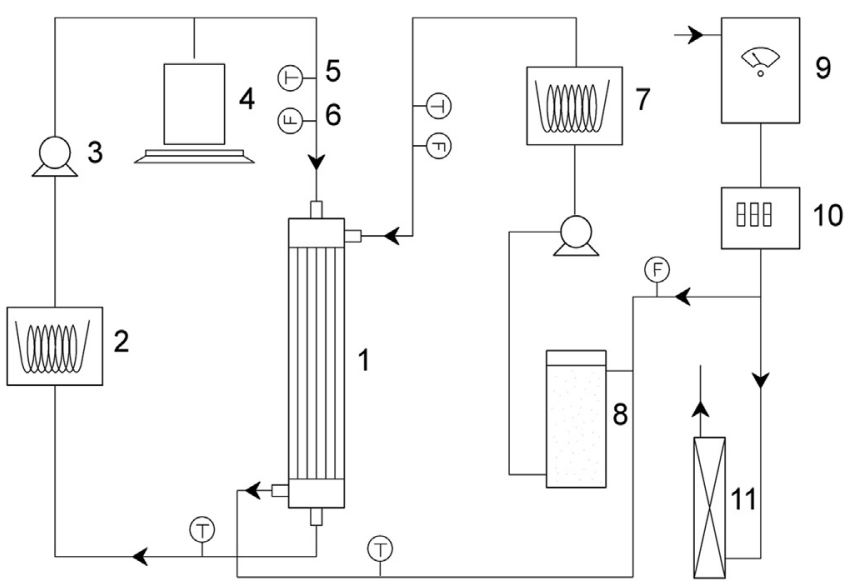

Fig. 1. Schematic diagram of the COMR system. (1) PVDF membrane module; (2) cryostat; (3) peristaltic pump; (4) water tank and balance; (5) thermometer; (6) flow meter; (7) thermostat bath; (8) glass reactor; (9) ozone generator; (10) ozone concentration analyzer; (11) KI wash bottle. 
and a permeate water circulation system.

During the experiment, the hot feed $(333 \mathrm{~K})$ and cold distillate water $(283 \mathrm{~K})$ flowed into the membrane module in a co-current mode, with a feed flow velocity of $0.038 \mathrm{~m} / \mathrm{s}$ and a distillate flow velocity of $0.354 \mathrm{~m} / \mathrm{s}$.

Ozone was produced by an ozone generator (Tonglin High-tech 3S-A3, Beijing, China) with oxygen supplied from a gas cylinder. An ozone detector (Ideal-2000, Zibo, China) and a flow meter were employed to maintain the ozone concentration at $60 \mathrm{mg} / \mathrm{L}$ and flow rate of $0.5 \mathrm{~L} / \mathrm{min}$. Ozone was introduced into the reactor using a Venturi jet made from PVDF material. The temperature of the glass reactor where the ozonation took place was set at $327 \mathrm{~K}$.

The catalysts screened in this work included: $\mathrm{Ce}(\mathrm{III}), \mathrm{Pb}(\mathrm{II})$, $\mathrm{Cd}(\mathrm{II}), \mathrm{Zn}(\mathrm{II}), \mathrm{Cu}(\mathrm{II}), \mathrm{Ni}(\mathrm{II}), \mathrm{Fe}(\mathrm{II}), \mathrm{Fe}(\mathrm{III})$ and $\mathrm{Cr}(\mathrm{III})$ and the chemical reagents were $\mathrm{CeCl}_{3} \cdot 7 \mathrm{H}_{2} \mathrm{O}, \quad \mathrm{Pb}\left(\mathrm{NO}_{3}\right)_{2}, \quad \mathrm{Cd}\left(\mathrm{NO}_{3}\right)_{2} \cdot 4 \mathrm{H}_{2} \mathrm{O}$, $\mathrm{Zn}\left(\mathrm{NO}_{3}\right)_{2} \cdot 6 \mathrm{H}_{2} \mathrm{O}, \quad \mathrm{CuCl}_{2} \cdot 2 \mathrm{H}_{2} \mathrm{O}, \quad \mathrm{Ni}\left(\mathrm{NO}_{3}\right)_{2} \cdot 6 \mathrm{H}_{2} \mathrm{O}, \quad \mathrm{FeCl}_{2} \cdot 4 \mathrm{H}_{2} \mathrm{O}$, $\mathrm{FeCl}_{3} \cdot 6 \mathrm{H}_{2} \mathrm{O}$ and $\mathrm{Cr}\left(\mathrm{NO}_{3}\right)_{3} \cdot 9 \mathrm{H}_{2} \mathrm{O}$, respectively. All the chemical reagents used in this work were purchased from Sinopharm with analytical grade without further purification.

\subsection{Analytical methods}

The mineralization degree of feed solution and the permeate water quality were evaluated by measuring the TOC concentration using a TOC analyzer (Shimadzu TOC L, Japan). The TOC was consist of KHP and KHP transformation products, such as ketones, carboxylic acids and aldehydes (Chen et al., 2008; Ho et al., 2012) The metal ion catalyst recovery rate and the salt rejection rate were assessed using an inductively coupled plasma mass spectrometry (ICP-MS, Agilent 7500a, USA). An electric conductivity monitor (CM-230A, Shijiazhuang Create Instrumentation Technologies, China) was also used to monitor the distillate water quality.

The functional groups presented on the membrane surface were detected using FT-IR spectroscopic method (Nicolet 8700, Thermo Fisher Scientific, USA). Membrane sample was dried and fixed with OMNI sampler. All spectra were recorded at $298 \mathrm{~K}$, and five samples were test for each membrane. The membrane topography was observed through scanning electro microscope (SEM) (S-3000N, Hitachi, Japan). Before observation, membrane samples were dried and sputtered with platinum using a Hitachi-1010 Ion Sputtering device at pressure lower than $10 \mathrm{kPa}$. The mechanical properties were tested with a tensiometer (Instron 5565-5 kN, Instron Corporation, USA) at room temperature. $50 \mathrm{~mm}$ membrane was used for test, and the sample was clamped at both ends and pulled at a constant rate of $10 \mathrm{~mm} / \mathrm{min}$.

\section{Results and discussion}

\subsection{Optimization of catalyst's type and loading}

Homogeneous catalyst promotes ozonation reaction through two major mechanisms: by generating free radicals or forming complexes (Nawrocki and Kasprzyk-Hordern, 2010), both of which were greatly affected by the reaction conditions. In order to determine the appropriate type of catalyst and the optimal loading, 9 metal ions and 5 concentrations were screened in this study. $\mathrm{Ce}(\mathrm{III}), \mathrm{Pb}(\mathrm{II}), \mathrm{Cd}(\mathrm{II}), \mathrm{Zn}(\mathrm{II}), \mathrm{Cu}(\mathrm{II}), \mathrm{Ni}(\mathrm{II}), \mathrm{Fe}(\mathrm{II}), \mathrm{Fe}(\mathrm{III})$, and $\mathrm{Cr}(\mathrm{III})$ were chosen as the catalyst candidates due to their excellent catalytic performance reported in prior literature (Wu et al., 2008). $0.5 \mathrm{mM}$ of metal ion was added into $400 \mathrm{~mL}$ solution containing $1000 \mathrm{mg} / \mathrm{L} \mathrm{KHP}$ and each experiment was ozonated for $30 \mathrm{~min}$. TOC was measured to evaluate the mineralization degree of the solution and the catalytic activity of the metal. The catalytic activity was quantified by enhancement factor, which was calculated according to the equation:
$E=\left(T O C_{0}^{C}-T O C_{30}^{C}\right) /\left(T O C_{0}^{O}-T O C_{30}^{O}\right)$. Where $E, T O C_{0}^{C}, T O C_{30}^{C}$, $T O C_{0}^{O}$ and $T O C_{30}^{O}$ were the enhancement factor, TOC with catalyst at 0 min, TOC with catalyst at $30 \mathrm{~min}$, TOC with ozone alone at 0 min and TOC with ozone alone at $30 \mathrm{~min}$, respectively. Each catalyst candidate was tested three times, and the average value was reported. Once the optimized catalyst was identified, different catalyst concentrations from 0.1 to $5 \mathrm{mM}$ were added into the system to identify the optimal loading. All the experiments were carried out at $327 \mathrm{~K}$.

Fig. 2A showed the enhancement factors of different catalysts. It was found that $\mathrm{Cu}(\mathrm{II})$ was the best catalyst among nine candidates, which displayed an enhancement index of $2.16 \pm 0.04$, suggesting the degradation of TOC with $\mathrm{Cu}$ (II) catalyst was more than two times better than the system with ozone alone. Among the metal ions, $\mathrm{Cu}(\mathrm{II}), \mathrm{Fe}(\mathrm{III})$ and $\mathrm{Fe}(\mathrm{II})$ showed excellent catalytic activity, while $\mathrm{Pb}(\mathrm{II}), \mathrm{Zn}$ (II) did not. Two major mechanisms worked in the homogeneous catalytic ozonation: generation free radicals and formation metal organic complexes (Kasprzyk-Hordern, 2003; Nawrocki and Kasprzyk-Hordern, 2010). The free radicals approach was affected by the reduction potential of the metals (Sánchez-Polo and Rivera-Utrilla, 2004), while the complexes approach may determine by the interaction of the metal and organic (Pines and Reckhow, 2002; Beltran et al., 2005). Besides, other factors like $\mathrm{pH}$ and reagents concentration may influence both the efficiency and the mechanism (Kasprzyk-Hordern, 2003). Therefore, the different performance for the catalysts may determine by the reaction system including metal and organic species and concentration, $\mathrm{pH}$ of the solution and etc. Both of the two mechanisms may contribute the catalytic ozonation process (Beltran et al., 2005).

Fig. 2B showed the effect of catalyst loading on the TOC removal efficiency. After $1 \mathrm{~h}$ ozonation, the remained TOCs were $75.7 \%$, $60.8 \%, 54.3 \%, 49.9 \%$ and $51.3 \%$ for the system with $0,0.1,0.5,1$, and $5 \mathrm{mM}$ catalyst loading respectively, which fitted perfectly to the pseudo-first-order kinetics model. The coefficient of determination $\left(\mathrm{R}^{2}\right)$ for all loadings were greater than 0.989 . Degradation rates $(\mathrm{k})$ were $0.00461,0.00825,0.01051,0.01161$ and $0.01122 \mathrm{~min}^{-1}$ for the systems with $0,0.1,0.5,1$ and $5 \mathrm{mM}$ catalyst loading, respectively. The highest ozonation rate was found at the catalyst loading of $1 \mathrm{mM}$. Further increasing the loading to $5 \mathrm{mM}$ did not promote the degradation rate. Catalyst may be overdose while more than $1 \mathrm{mM}$ metal ion added. Therefore $1 \mathrm{mM}$ was determined to be the optimal loading.

\subsection{Organic matter and salt removal efficiency in the hybrid process}

During the MD process, vapor molecules transported across the hydrophobic membrane under the thermal driving force while the salts were remained. Owing to the ozonation, KHP may degrade into ketones, carboxylic acids, aldehydes and carbon dioxide (Chen et al., 2008; Ho et al., 2012). The degradation products may also transport across the membrane because of their volatility, and therefore affect the permeate water quality. Through investigating the permeate water quality, the experiments aimed to exhibit the two major functions of the membrane: clean water production and catalyst separation. Firstly, the hybrid processes were operated with different feed $\mathrm{KHP}$ and $\mathrm{NaCl}$ concentrations. Three concentration levels of 200, 500 and $1000 \mathrm{mg} / \mathrm{L} \mathrm{KHP}$ and $\mathrm{NaCl}$ were operated for $5 \mathrm{~h}$. The permeate water TOC, as well as the ion concentrations were measured. In order to measure the catalytic efficiency of the recovered catalyst, a four-stage experiment was performed. In stage one, $800 \mathrm{~mL}$ of water containing $1000 \mathrm{mg} / \mathrm{L}$ KHP, $1000 \mathrm{mg} / \mathrm{L} \mathrm{NaCl}$ and $1 \mathrm{mM} \mathrm{Cu}(\mathrm{II})$ was ozonated and concentrated for $5 \mathrm{~h}$. Subsequently, in stage two, $300 \mathrm{~mL}$ water with 
A

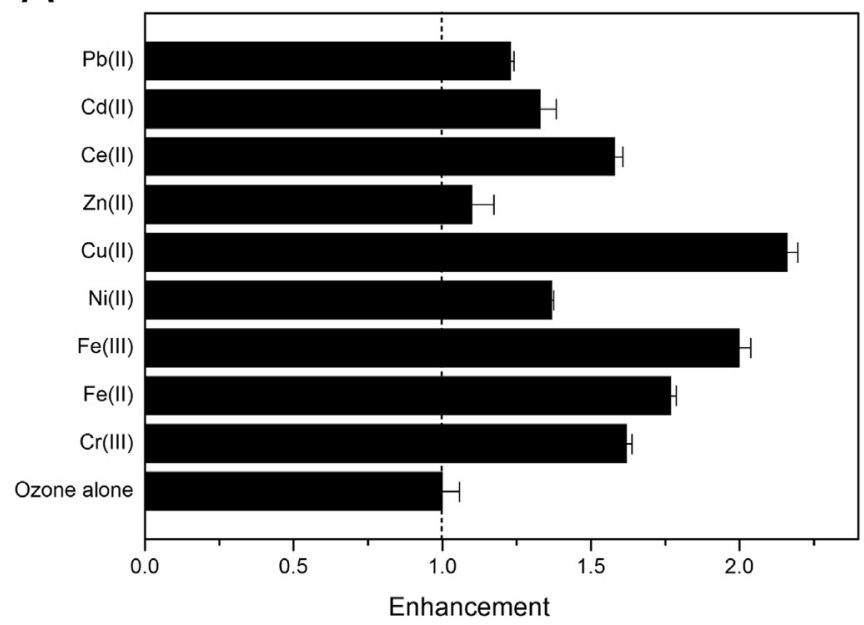

B

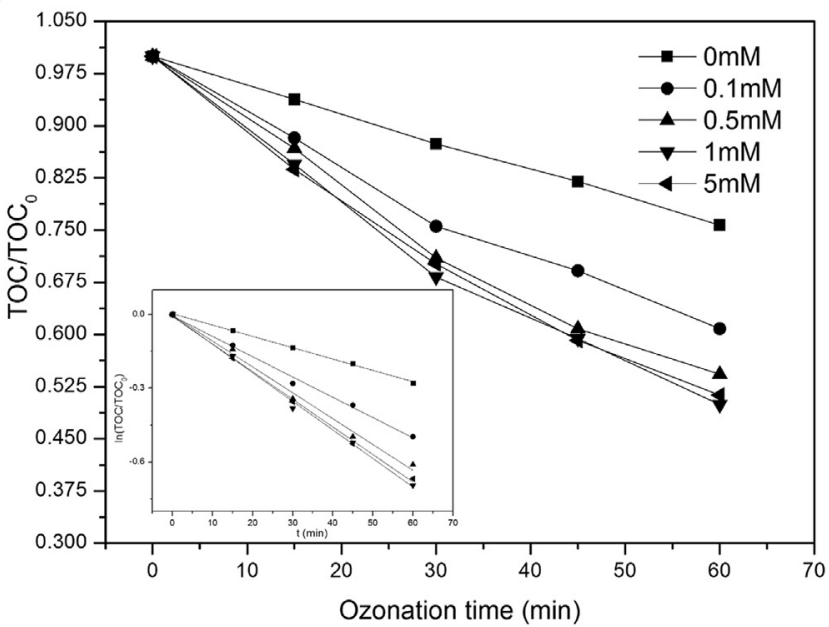

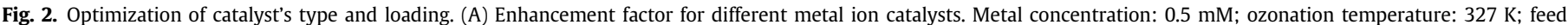

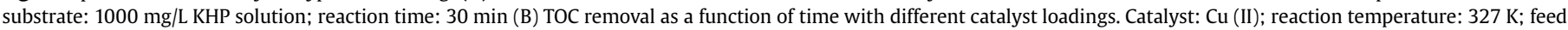
substrate: $1000 \mathrm{mg} / \mathrm{L} \mathrm{KHP} \mathrm{solution.}$

$1000 \mathrm{mg} / \mathrm{L} \mathrm{KHP}$ was added to the reactor to replenish the water and organic matter lost in stage one. The solution was ozonated and concentrated for another $5 \mathrm{~h}$. Stage three and four followed the same protocol as stage two. The ion concentration and the TOC of concentrate and permeate water were also measured. The TOC of concentrate water was measured directly, while the TOC of permeate water was calculated from the equation below based on material balance law:

$C_{p e r}=\left(\left(V_{0}+V_{d i s} T_{t}\right) C_{t}-\left(V_{0}+V_{d i s} T_{t-1}\right) C_{t-1}\right) / V_{d i s}$. Where $C_{p e r}$, $V_{0}, V_{d i s}, T_{t}, T_{t-1}, C_{t}$ and $C_{t-1}$ were the TOC of permeate water at time $t$, water volume of permeate side, volume of permeate water during the test period, time at the end of experiment, time at the beginning of experiment, TOC of the permeate side water at the end of experiment, and TOC of permeate side water at the beginning of experiment, respectively. $V_{0}$ was $420 \mathrm{~mL}$ and $V_{\text {dis }}$ was $68.1 \mathrm{~mL}$ in the experiment. $C_{p e r}, C_{t}$ and $C_{t-1}$ were assumed to be constant between two samples for the sake of simplification.

Results of water quality for different feed concentrations are showed in Fig. 3A. The TOC of the permeate water ranged from 5.71 to $15.97 \mathrm{mg} / \mathrm{L}$. It should be noted that the excessive amount of KHP was used in this work which contributed to a higher level of TOC in the permeate stream. In terms of the rejection rate, it ranged from $88.2 \%$ to $98.6 \%$. The highest rejection rate was detected at the first hour with $1000 \mathrm{mg} / \mathrm{L} \mathrm{KHP}$ in the feed solution, when the concentration ratio between KHP and its decomposition substrates may be the lowest.

As showed in Fig. 3B. The concentration of Na was $116 \mu \mathrm{g} / \mathrm{L}$ and nearly no $\mathrm{Cu}$ was detected at the initial of permeate side water. As clean water crossed the membrane, Na was reduced from 116 to $60 \mu \mathrm{g} / \mathrm{L}$ as the dilution effect. The concentrations of $\mathrm{Cu}$ were less than $5 \mu \mathrm{g} / \mathrm{L}$ in all the experiments. Clearly, the metal ion catalysts were almost completely recovered by membrane distillation. In principle, no metal ions should transport across the membrane. However, some ions did permeate through the membrane in practice, although the amount was negligible. Membrane wetting may be the reason for metal transporting across the membrane. As previous study, wetting phenomenon, especially partial wetting always occurred in membrane distillation and membrane

\section{A}

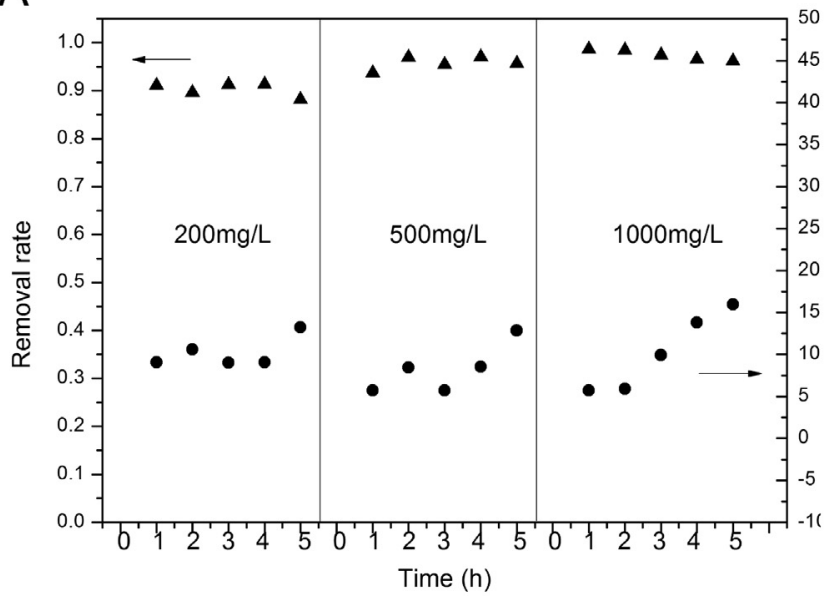

B

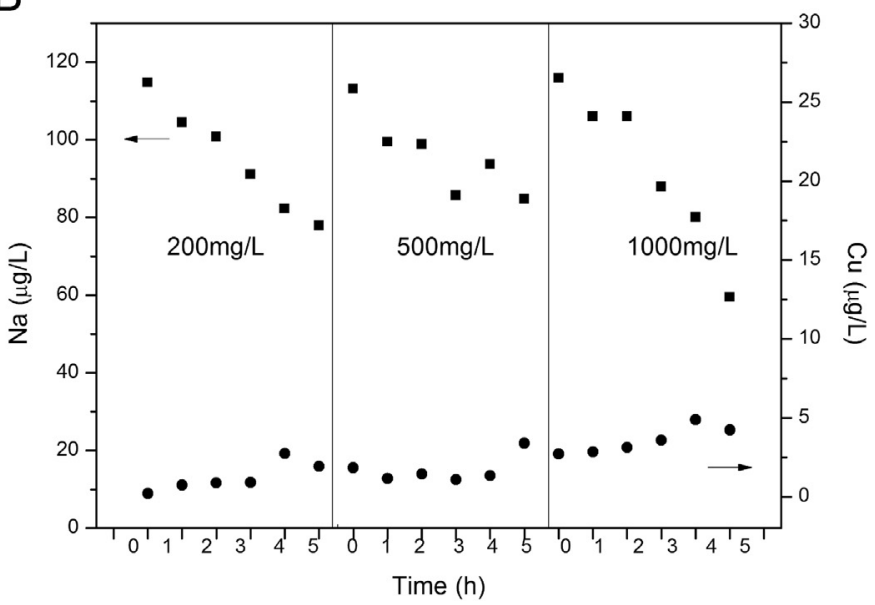

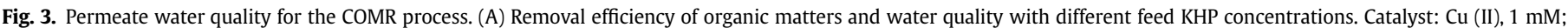

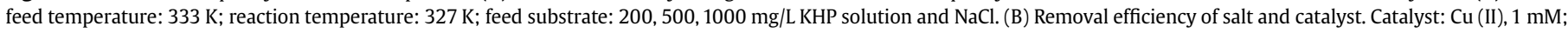
feed temperature: $333 \mathrm{~K}$; reaction temperature: $327 \mathrm{~K}$; feed substrate: 200, 500, $1000 \mathrm{mg} / \mathrm{L} \mathrm{KHP}$ solution and $\mathrm{NaCl}$. 
contactors (Ge et al., 2014; Mosadegh-Sedghi et al., 2014; GuillenBurrieza et al., 2016). In this experiment, the possibility can be even higher in consideration of the high organic concentration of feed water (Lv et al., 2010). While the membrane was partial wetted, the non-volatile metal ions crossed the membrane through the wetted pores. As the concentration of metal ions in the permeate water were low, the wetting degree of the membrane should be very small.

In Fig. 4, changes of TOC concentrations in the permeate and feed water streams during the four-stage experiment is presented. It was observed that TOC increased in both permeate and feed waters. In the permeate water, TOC mainly included the volatile degradation products of KHP. A clear trend of increased TOC concentration can be found throughout the four-stage experiment, suggesting the permeation rate of volatile substrates increased over time. As reported in literature, KHP was first ozonated to large intermediates with molecular structure similar to KHP (Intermediate 1 , I1), then it further broke down to ketones, aldehydes and carboxylic acids (Intermediate2, I2) (Ho et al., 2012). I2 was much more volatile than I1 and KHP. As a result, a large number of 12 transported may across the membrane. The TOC concentration of the permeate water increased linearly with a slope of 2.212 and $\mathrm{R}^{2}$ of 0.9608 . The TOC concentration was found to be $46.08 \mathrm{mg} / \mathrm{L}$ at the end of stage four. Although it was still relatively high, the result was acceptable considering the TOC in the feed side was $1010.5 \mathrm{mg} / \mathrm{L}$. Throughout all the four stages, the TOC rejection ranged between 95.2\% and $99.6 \%$.

In terms of the concentrated water, non-volatile substances may enriched due to the distillation effect, including $\mathrm{NaCl}, \mathrm{Cu}(\mathrm{II}), \mathrm{KHP}$ and some degradation products of KHP. These results clearly revealed that the ozonation rate was slower than the concentrate rate. In stage one, $30.35 \%$ water was distillated while only $17.75 \%$ TOC was removed by ozone. As a result, the TOC in the concentrated water continuously increased with time. At the end of each stage, TOC decreased as water containing less organic substrate was added. Due to the large number of organic substrates (more than 10 different types) presented in the reactor and the different concentration of every substrate at the beginning of each stage, the catalytic efficiency can not be calculated from the TOC degradation rate. $68,88,115$, and $126 \mathrm{mg}$ TOC was removed through catalytic ozonation process in four different stage experiments, and the TOC removal rate was $17.7 \%, 18.7 \%, 22.1 \%$ and $22.7 \%$, respectively. The TOC removal rate improved as the substrate concentration increased.

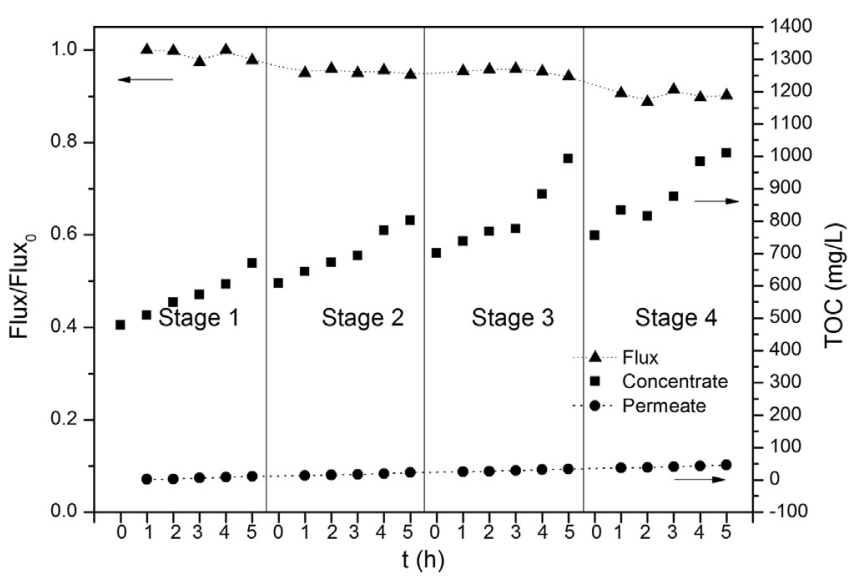

Fig. 4. Permeate and feed water TOC for a four-stage experiment. Catalyst: $\mathrm{Cu}$ (II), $1 \mathrm{mM}$; feed temperature: $333 \mathrm{~K}$; reaction temperature: $327 \mathrm{~K}$; feed substrate: $1000 \mathrm{mg} / \mathrm{L} \mathrm{KHP}$ solution and $\mathrm{NaCl}$.
These results clearly demonstrated that the hybrid process proposed in this work offered an efficient approach for saline organic wastewater treatment, with organic substrates, salts and catalysts being removed simultaneously. More specifically, salts and ionic catalysts were almost completely removed and $88.2 \%-$ 98.6\% organic substrates were removed depending on the feed concentration. The permeate water quality was largely dependent on the ozonation time. When increasing the TOC of feed water to $1000 \mathrm{mg} / \mathrm{L}$, less than $16 \mathrm{mg} / \mathrm{L}$ of TOC was found in the permeate water after $5 \mathrm{~h}$ of operation. In addition, the results also demonstrated excellent stability of the catalysts, as no sign of decrease in catalytic activity was observed after the four consecutive stages.

\subsection{Effect of ozonation on membrane fouling mitigation}

In this work, the membrane flux was tested during $60 \mathrm{~h}$ of operation to investigate the fouling phenomenon. Fig. 5 showed the flux and conductivity of both membrane distillation and the hybrid process. For membrane distillation, the flux declined by $39.6 \%$ at the end of $60 \mathrm{~h}$, whilst in the case of the hybrid system, only 9.5\% flux decline was observed. Membrane flux decline was mainly attributed to the membrane fouling that caused by the accumulation of deposits on membrane surface or in membrane pores. In Fig. 6, the deposits on the membrane surface were visualized. A large number of deposits can be found on the membrane surface after $60 \mathrm{~h}$ membrane distillation (image B). For the hybrid system, much fewer deposits were found on the membrane surface after $20 \mathrm{~h}$ and $60 \mathrm{~h}$ of operation. Furthermore, it was also observed that the size of deposits were much smaller in the case of hybrid process.

These results clearly revealed that the membrane fouling was prominently minimized in the hybrid process. The interaction between foulants and membrane surface affected the formation of deposits. The two major factors affecting the reaction were foulant concentration and hydrophobicity (Tijing et al., 2015). Organic concentration was much higher in the MD process than the hybrid one. At the end of operation, TOC in the MD process was $1097 \mathrm{mg} / \mathrm{L}$ while only $610 \mathrm{mg} / \mathrm{L}$ was found in the hybrid one. In addition, during the hybrid process, KHP may ozonated to more hydrophilic substrates. As a result, the combination between foulants and hydrophobic PVDF membrane surface was weaker, and the fouling behavior was therefore mitigated.

In terms of conductivity, it decreased for both cases at first few

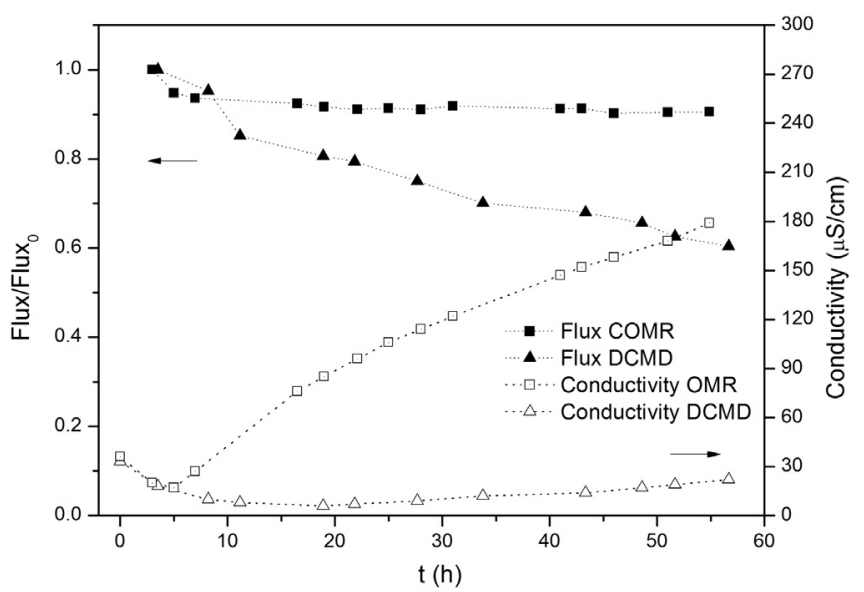

Fig. 5. Flux and conductivity of COMR and DCMD processes. Catalyst: $\mathrm{Cu}$ (II), $1 \mathrm{mM}$ feed temperature: $333 \mathrm{~K}$; reaction temperature: $327 \mathrm{~K}$; feed substrate: $1000 \mathrm{mg} / \mathrm{L} \mathrm{KHP}$ solution and $\mathrm{NaCl}$; volume: $10 \mathrm{~L}$ 

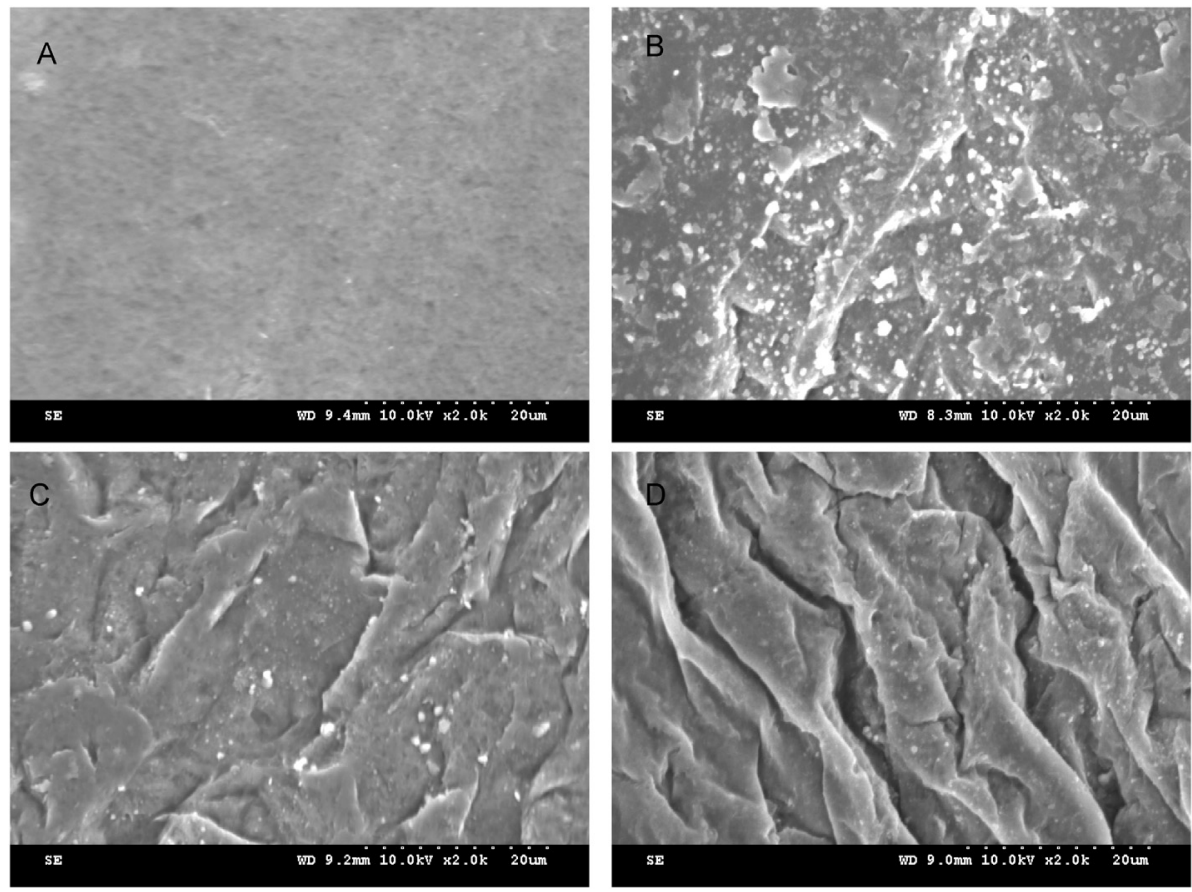

Fig. 6. SEM images of membrane surfaces. (A) original membrane, (B) membrane after $60 \mathrm{~h} \mathrm{DCMD,} \mathrm{(C)} \mathrm{membrane} \mathrm{after} 20$ h COMR, and (D) membrane after $60 \mathrm{~h}$ COMR.

hours and then increased, with a much higher level of increment observed in the case of COMR process. Small degree of membrane wetting may occurred in the DCMD process, as a result, KHP transported across the membrane pores through the wetting pores. While in the hybrid process, KHP degradation substrates (such as low-molecular-weight ketones and acids) passed through the membrane pores by evaporation mainly (Chen et al., 2008; Ho et al., 2012). Formic acid, acetic acid and oxalic acid were detected in the permeate water. As we can see in Fig. S2, three carboxylic acids were increased with time. The tendency was in accordance with the conductivity, which indicated volatile KHP degradation products crossing the membrane. In the COMR process, the conductivity was eight time higher than that in the DCMD process. As aforementioned, only marginal amount of salts crossed the membrane, therefore the increased conductivity in the case of COMR process was mainly due to the permeation of KHP degradation substrates.

\subsection{Stability of the PVDF hollow fiber membrane}

The PVDF membranes were subject to morphology, mechanical property, hydrophobicity and surface group analyses to evaluate the membrane stability.

The SEM images of the membranes were showed in Fig. 6. The surface was smooth for the original membrane before operation (image $\mathrm{A}$ ) and the membrane after $60 \mathrm{~h}$ distillation process showed nearly the same morphology except for the foulant deposits presented on the surface (image B). Wrinkles were observed on the membrane surface after the COMR hybrid process (images $C$ and $D$ ), and these wrinkles became more obvious with longer ozonation treatment. Such an observation can be ascribed to the diffusion of solution into the polymer matrix that caused the membrane swelling (Wang et al., 2014). In contrast, no swelling phenomenon was found on membrane surface after $60 \mathrm{~h}$ DCMD process. Membrane swelling was dependent with liquid entry pressure (LEPw) according to Laplace-Young equation (Lv et al., 2010; Wang et al., 2014) which was determined by contact angle between membrane surface and water. As we can see from Fig. S3 the contact angle decreased from $105.9^{\circ}$ to $97.4^{\circ}$ and $77.0^{\circ}$, after 20 and $60 \mathrm{~h}$ COMR process as the ozonation effect. Membrane wetting may occurred and water was much easier to enter the membrane matrix and the deformation was more severe. These results suggested that ozonation promote the swelling process, which due to the less hydrophobic surface after ozonation. As a result, the membrane was easier to swell. These change in membrane surface after $60 \mathrm{~h}$ ozonation may affect the performance of the hybrid process.

The mechanical properties were also investigated using a tensiometer and the results are presented in Fig. 7A. Stress-strain curves of the four hollow fiber membranes were presented. The breaking force ranged from 151 to $161 \mathrm{cN}$, and the elongation ranged from $215 \%$ to $224 \%$. The results indicated the membrane mechanical properties were maintained after $60 \mathrm{~h}$ ozonation.

The surface groups were analyzed using FT-IR. As showed in Fig. 7B, all the spectra appeared to be almost identical for the four different hollow fiber membranes except for a new peak appearing at about $1588 \mathrm{~cm}^{-1}$, which was related to the KHP that deposited on the membrane surface. For the PVDF membrane, hydroxyl and carbonyl groups may appear on the surface after ozonation treatment. However, it was not evidenced in this work, which might be due to the limited number of these groups to be detected by the FTIR.

From the results of mechanical properties and FT-IR spectra, the PVDF membrane showed good durability after $60 \mathrm{~h}$ operation. However, swelling phenomenon was found on membrane surface, which indicated the risk of membrane degradation. The PVDF membrane is suitable for a limited period of experiment. However, more tests are needed for a long time application.

\section{Conclusions}

In this work a novel hybrid membrane reactor combining homogeneous catalytic ozonation and membrane distillation was developed. The organic matter and salt removal efficiency, the effect of ozonation on membrane fouling mitigation and stability of PVDF hollow fiber membranes were discussed. Homogeneous 
A

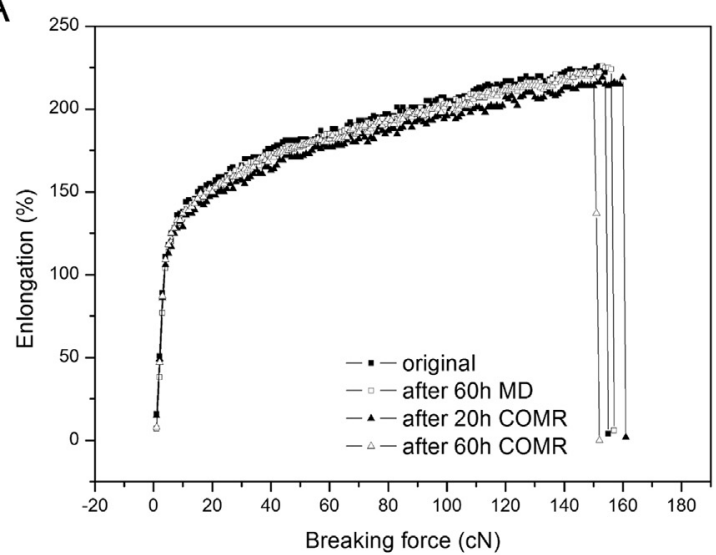

B

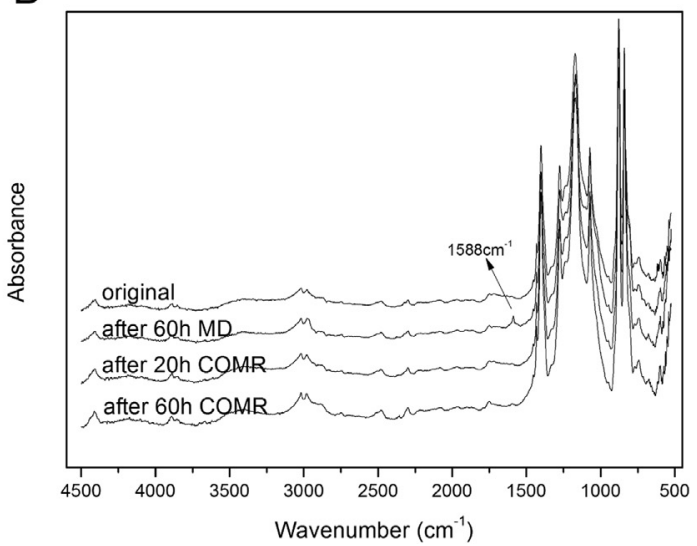

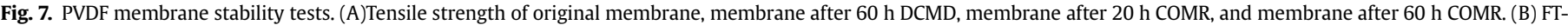
IR spectra of original membrane, membrane after $60 \mathrm{~h}$ DCMD, membrane after $20 \mathrm{~h}$ COMR, and membrane after $60 \mathrm{~h}$ COMR.

catalyst was found almost fully recovered by the membrane distillation process, which was critical when using expensive or poisonous catalysts. For saline organic wastewater, salts and organic matters were simultaneously removed in this simple hybrid reactor. For $5 \mathrm{~h}$ operation and $1000 \mathrm{mg} / \mathrm{L} \mathrm{KHP} \mathrm{feed} \mathrm{solution,}$ more than 96\% TOC was removed and the permeate water was less than $16 \mathrm{mg} / \mathrm{L}$. Meanwhile, membrane fouling was also mitigated. The flux only decreased by $9.5 \%$ after $60 \mathrm{~h}$ operation owing to the ozonation. The membrane durability tests showed that the PVDF membrane was stable for the testing period.

\section{Acknowledgement}

This work was supported by the National Science and Technology Major Project (No. 2012ZX07202006-003) and the National Natural Science Foundation of China (Grant NO. 51378491 and 51578533).

\section{Appendix A. Supplementary data}

Supplementary data related to this article can be found at http:// dx.doi.org/10.1016/j.chemosphere.2016.06.070.

\section{References}

Beltrán, F.J., 2004. Ozone Reaction Kinetics for Water and Wastewater Systems. Taylor \& Francis Group LLC, Boca Raton.

Beltran, F.J., Rivas, F.J., Montero-de-Espinosa, R., 2005. Iron type catalysts for the ozonation of oxalic acid in water. Water Res. 39, 3553-3564.

Benotti, M.J., Stanford, B.D., Wert, E.C., Snyder, S.A., 2009. Evaluation of a photocatalytic reactor membrane pilot system for the removal of pharmaceuticals and endocrine disrupting compounds from water. Water Res. 43, 1513-1522.

Buffle, M.O., Schumacher, J., Salhi, E., Jekel, M., von Gunten, U., 2006. Measurement of the initial phase of ozone decomposition in water and wastewater by means of a continuous quench-flow system: application to disinfection and pharmaceutical oxidation. Water Res. 40, 1884-1894.

Chen, Y.H., Shang, N.C., Hsieh, D.C., 2008. Decomposition of dimethyl phthalate in an aqueous solution by ozonation with high silica zeolites and UV radiation. J. Hazard. Mater. 157, 260-268.

Choo, K.-H., Tao, R., Kim, M.-J., 2008. Use of a photocatalytic membrane reactor for the removal of natural organic matter in water: effect of photoinduced desorption and ferrihydrite adsorption. J. Membr. Sci. 322, 368-374.

El-Bourawi, M.S., Ding, Z., Ma, R., Khayet, M., 2006. A framework for better understanding membrane distillation separation process. J. Membr. Sci. 285, 4-29.

Ge, J., Peng, Y., Li, Z., Chen, P., Wang, S., 2014. Membrane fouling and wetting in a DCMD process for RO brine concentration. Desalination 344, 97-107.

Gottschalk, C., Libra, J.A., Saupe, A., 2000. Ozonation of Water and Waste Water: a Practical Guide to Understanding Ozone and Its Applications. WILEY-VCH Verlag GmbH \& Co. KGaA., Weinheim.

Guillen-Burrieza, E., Mavukkandy, M.O., Bilad, M.R., Arafat, H.A., 2016. Understanding wetting phenomena in membrane distillation and how operational parameters can affect it. J. Membr. Sci. 515, 163-174.

Heng, S., Yeung, K.L., Djafer, M., Schrotter, J.-C., 2007. A novel membrane reactor for ozone water treatment. J. Membr. Sci. 289, 67-75.

Ho, H.L., Chan, W.K., Blondy, A., Yeung, K.L., Schrotter, J.-C., 2012. Experiment and modeling of advanced ozone membrane reactor for treatment of organic endocrine disrupting pollutants in water. Catal. Today 193,120-127.

Hou, D., Wang, J., Sun, X., Ji, Z., Luan, Z., 2012. Preparation and properties of PVDF composite hollow fiber membranes for desalination through direct contact membrane distillation. J. Membr. Sci. 405-406, 185-200.

Kasprzyk-Hordern, B., 2003. Catalytic ozonation and methods of enhancing molecular ozone reactions in water treatment. Appl. Catal. B 46, 639-669.

Kit Chan, W., Jouët, J., Heng, S., Lun Yeung, K., Schrotter, J.-C., 2012. Membrane contactor/separator for an advanced ozone membrane reactor for treatment of recalcitrant organic pollutants in water. J. Solid State Chem. 189, 96-100.

Leong, S., Razmjou, A., Wang, K., Hapgood, K., Zhang, X., Wang, H., 2014. TiO2 based photocatalytic membranes: a review. J. Membr. Sci. 472, 167-184.

Lv, Y., Yu, X., Tu, S.-T., Yan, J., Dahlquist, E., 2010. Wetting of polypropylene hollow fiber membrane contactors. J. Membr. Sci. 362, 444-452.

Mosadegh-Sedghi, S., Rodrigue, D., Brisson, J., Iliuta, M.C., 2014. Wetting phenomenon in membrane contactors - causes and prevention. J. Membr. Sci. 452, $332-353$.

Mozia, S., 2010. Photocatalytic membrane reactors (PMRs) in water and wastewater treatment. A review. Sep. Purif. Technol. 73, 71-91.

Mozia, S., Darowna, D., Szymański, K., Grondzewska, S., Borchert, K., Wróbel, R., Morawski, A.W., 2014. Performance of two photocatalytic membrane reactors for treatment of primary and secondary effluents. Catal. Today 236, 135-145.

Mozia, S., Morawski, A.W., 2006. Hybridization of photocatalysis and membrane distillation for purification of wastewater. Catal. Today 118, 181-188.

Mozia, S., Morawski, A.W., Toyoda, M., Tsumura, T., 2009. Effect of process parameters on photodegradation of Acid Yellow 36 in a hybrid photocatalysis-membrane distillation system. Chem. Eng. J. 150, 152-159.

Mozia, S., Morawski, A.W., Toyoda, M., Tsumura, T., 2010. Integration of photocatalysis and membrane distillation for removal of mono- and poly-azo dyes from water. Desalination 250, 666-672.

Mozia, S., Tomaszewska, M., Morawski, A.W., 2006. Removal of azo-dye Acid Red 18 in two hybrid membrane systems employing a photodegradation process. Desalination 198, 183-190.

Nawrocki, J., Kasprzyk-Hordern, B., 2010. The efficiency and mechanisms of catalytic ozonation. Appl. Catal. B 99, 27-42.

Pines, D.S., Reckhow, D.A., 2002. Effect of dissolved cobalt(II) on the ozonation of oxalic acid. Environ. Sci. Technol. 36, 4046-4051.

Qu, D., Qiang, Z., Xiao, S., Liu, Q., Lei, Y., Zhou, T., 2014. Degradation of Reactive Black 5 in a submerged photocatalytic membrane distillation reactor with microwave electrodeless lamps as light source. Sep. Purif. Technol. 122, 54-59.

Sánchez-Polo, M., Rivera-Utrilla, J., 2004. Ozonation of 1,3,6-naphthalenetrisulfonic acid in presence of heavy metals. J. Chem. Technol. Biotechnol. 79, 902-909.

Shim, W.G., He, K., Gray, S., Moon, I.S., 2015. Solar energy assisted direct contact membrane distillation (DCMD) process for seawater desalination. Sep. Purif. Technol. 143, 94-104.

Tijing, L.D., Woo, Y.C., Choi, J.S., Lee, S., Kim, S.H., Shon, H.K., 2015. Fouling and its control in membrane distillation-A review. J. Membr. Sci. 475, 215-244.

Wachinski, A.M., 2013. Membrane Process for Water Reuse. McGraw-Hill, New York.

Wang, Z., Fang, M., Ma, Q., Yu, H., Wei, C.-C., Luo, Z., 2014. Investigation of membrane wetting in different absorbents at elevated temperature for carbon dioxide capture. J. Membr. Sci. 455, 219-228.

Wu, C.H., Kuo, C.Y., Chang, C.L., 2008. Homogeneous catalytic ozonation of C.I Reactive Red 2 by metallic ions in a bubble column reactor. J. Hazard. Mater. 154 $748-755$. 